\title{
Structural, vibrational and electronic properties of cis and trans conformers of 4-hydroxy-l-proline: a density functional approach
}

\author{
Ambrish K. Srivastava ${ }^{a}$, Anoop K. Pandey ${ }^{b}$, Shashi K. Gangwar ${ }^{a}$, \\ and Neeraj Misra ${ }^{a *}$ \\ ${ }^{a}$ Department of Physics, University of Lucknow, Lucknow, Uttar Pradesh- 226007, \\ India \\ ${ }^{b}$ Department of Physics, Govt. D.P.G. College, Dantewada, Chhattishgarh-494449, \\ India
}

Received 22 February 2014; Accepted (in revised version) 18 April 2014

Published Online 29 October 2014

\begin{abstract}
. 4-hydroxy-l-proline is formed by hydroxylation of proline, an amino acid found in protein, whose inhibition results in hair problems in human, causing scurvy disease. We report a theoretical study on cis and trans conformers of 4-hydroxy-lproline using first principle density functional approach at B3LYP $/ 6-31+G(d, p)$ level. The equilibrium structures of both conformers are obtained to analyze their vibrational properties. The calculated vibrational modes are assigned and interpreted on the basis of potential energy distribution analysis. A good correlation has been obtained between calculated frequencies and corresponding experimental values from FTIR spectra. The electronic properties of both conformers are also calculated and discussed.
\end{abstract}

PACS: 31.15.A, 31.15.es, 31.15.V

Key words: 4-hydroxy-l-proline, cis-trans conformers, vibrational analysis, density functional theory

\section{Introduction}

4-hydroxyproline or 4-hydroxy-l-proline (4HLP) is a non native amino acid which differs from proline, a proteinogenic amino acid, by the presence of a hydroxyl $(-\mathrm{OH})$ group attached to the gamma carbon. It was isolated from hydrolyzed gelatin by Hermann Emil in the beginning of 20th century. Although, it is not directly incorporated into proteins, it comprises about $4 \%$ of all amino acids found in animal tissue, an amount greater than

*Corresponding author. Email address: neerajmisra11@gmail.com (N. Misra) 
many other translationally incorporated amino acids [1]. Furthermore, hydroxyproline is a major component of the protein collagen [2] which plays key roles in collagen stability [3], permitting the sharp twisting of the collagen helix [4]. For this reason, its content has been used as an indicator to determine collagen and/or gelatin amount. Moreover, hydroxyproline rich glycoproteins are also found in plant cell walls [5].

The pharmacological impact of this non-proteinogenic amino acid can never be ignored. Since, the hydroxylation of proline requires ascorbic acid i.e. vitamin $C$, the absence of vitamin $C$ in humans inhibits the hydroxylation, consequently reducing the stability of collagen molecule and causing the hair problems i.e. scurvy. Apart from this, the increased serum and urine levels of hydroxyproline have also been demonstrated in Paget's disease [6]. Quantum chemical methods provide a lot of information about the system of biological interests that often complements with the experiment findings. Many chemical and / or biological properties are closely associated to the molecular structures or geometries. Different conformations of the same molecule may lead to different chemical properties. The aim of the present study includes detailed structural and vibrational analyses of two potential conformers of 4HLP, namely, cis-4HLP and trans-4HLP. Our literature survey reveals a recent study performed on trans-4HLP [7], however, there is no corresponding study on cis-4HLP to the best of our knowledge. Thus, the present study provides a comparative analysis of cis and trans conformers of 4HLP for the first time.

\section{Computational methods}

Our quantum chemical calculations are based density functional scheme as implemented in Gaussian 09 package [8]. Initial geometries of cis- and trans-4HLYP are fully optimized without any symmetry constraint in the potential energy surfaces (PESs) using gradient corrected hybrid functional B3LYP $[9,10]$ and $6-31+G(d, p)$ basis set. B3LYP is one of the most widely used functionals in case of medium size biomolecules and hence, employed successfully in a number of previous studies [7, 11-14]. Vibrational frequency calculations are repeated using optimized geometries at the same level of theory. All calculated frequencies are found to be real which ensure that the optimized geometries belong to true minima on respective PESs.

Normal modes of both conformers of 4HLP are interpreted and assigned in terms of potential energy distribution (PED) using Gar2ped program [15]. The calculated frequencies are uniformly scaled by a factor of 0.9648 [16] in order to compensate for anharmonicity of vibrations as well as electron correlations. The scaled frequencies are compared with corresponding experimental FTIR values. The FTIR spectra of cis-4HLP and trans-4HLP are adopted from literature, which are available from SDBS website [17]. 


\section{Results and discussion}

\subsection{Structural properties}

The optimized geometries of cis- and trans-4HLP are displayed in Fig. 1 and corresponding structural parameters (bond-lengths and bond-angles) are listed in Table 1. 4HLP is composed of one hetero-pentagonal ring with one carbon replaced by nitrogen. Pentagonal ring deviates from planarity due to repulsion created by non-bonding electrons of nitrogen. The covalent bond between the side-chain and nitrogen backbone has relevant structural consequences on the properties of both conformers. However, there is a strict dependence between main- and side-chain proline conformations. This is reflected in the non-bonding distances between backbone atoms, $\mathrm{R}(1-4)$ which is $2.12 \AA$ for cis and 2.118 $\AA$ for trans and $\mathrm{R}(1-5), 2.128 \AA$ (cis) and $2.12 \AA$ (trans) (not given in Table 1). The observed correlation between proline puckering and peptide bond conformation suggests a new mechanism to explain the reported shift of the cis-trans equilibrium in proline derivatives.

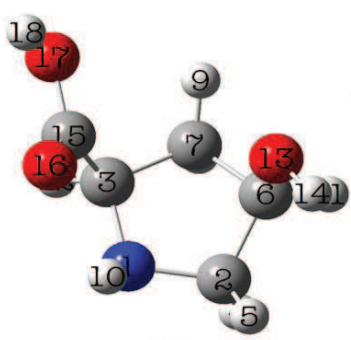

(a)

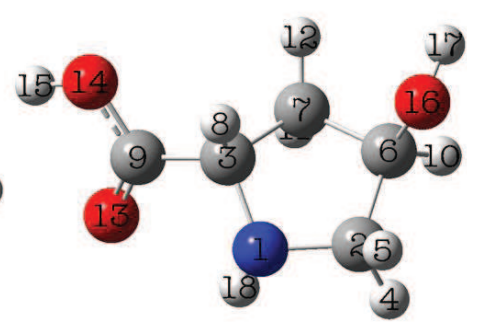

(b)

Figure 1: Optimized geometries of 4-hydroxy-l-proline at B3LYP/6-31+G(d,p) level (a) cis conformer (b) trans conformer. Dark, light, red and blue spheres represent $\mathrm{C}, \mathrm{H}, \mathrm{O}$ and $\mathrm{N}$ atoms, respectively.

In cis and trans conformers the same group (-OH and $-\mathrm{COOH})$ are present at different angular positions of hetero-pentagonal ring. This results in the change in ring geometries which have also been proven to be a sensitive indicator of the interaction between the substituent and the ring. Consequently, a re-adjustment takes place in the ring due to presence of these groups in both cis and trans conformations. The average bond-length of $\mathrm{C}-\mathrm{C}$ in ring for cis (1.52 $\AA$ ) takes slightly lower value that in trans-4HLP $(1.53 \AA)$. However $\mathrm{C}-\mathrm{N}$ bond-length in cis $(1.47 \AA)$ becomes slightly higher than that in trans conformer, $1.46 \AA$ (see Table 1). In general, calculated bond-lengths are consistent with the observed values for 4HLP [18] by X-ray and neutron diffraction. For instance, observed C-C bond-lengths in 4HLP are found to be in between $1.52 \AA$ and $1.53 \AA$.

\subsection{Vibrational properties}

In this section, we discuss the vibrational properties of cis- and trans-4HLP. The calculated frequencies are scaled as mentioned earlier and compared with corresponding FTIR 
Table 1: Bond-length $(R$ in $\AA)$ and bond-angle $\left(A\right.$ in $\left.{ }^{\circ}\right)$ of cis- and trans-4-hydroxy- $l$-proline calculated at B3LYP/6-31+G(d,p) level. Refer to Fig. 1 for atomic labeling.

\begin{tabular}{|c|c|c|c|}
\hline \multicolumn{2}{|c|}{ cis-4-hydroxy-l-proline } & \multicolumn{2}{|c|}{ trans-4-hydroxy-1-proline } \\
\hline Parameter & Value & Parameter & Value \\
\hline$R(1-2)$ & 1.475 & $R(1-2)$ & 1.475 \\
\hline$R(1-3)$ & 1.469 & $R(1-3)$ & 1.462 \\
\hline$R(1-10)$ & 1.016 & $R(1-18)$ & 1.017 \\
\hline$R(2-4)$ & 1.099 & $R(2-4)$ & 1.094 \\
\hline$R(2-5)$ & 1.096 & $R(2-5)$ & 1.095 \\
\hline$R(2-6)$ & 1.537 & $R(2-6)$ & 1.553 \\
\hline$R(3-7)$ & 1.555 & $R(3-7)$ & 1.555 \\
\hline$R(3-12)$ & 1.099 & $R(3-8)$ & 1.096 \\
\hline$R(3-15)$ & 1.525 & $R(3-9)$ & 1.512 \\
\hline$R(6-7)$ & 1.528 & $R(6-7)$ & 1.539 \\
\hline$R(6-11)$ & 1.099 & $R(6-10)$ & 1.098 \\
\hline$R(6-13)$ & 1.432 & $R(6-16)$ & 1.434 \\
\hline$R(7-8)$ & 1.094 & $R(7-11)$ & 1.097 \\
\hline$R(7-9)$ & 1.091 & $R(7-12)$ & 1.094 \\
\hline$R(13-14)$ & 0.966 & $R(9-13)$ & 1.214 \\
\hline$R(15-16)$ & 1.213 & $R(9-14)$ & 1.354 \\
\hline$R(15-17)$ & 1.354 & $R(14-15)$ & 0.973 \\
\hline$R(17-18)$ & 0.972 & $R(16-17)$ & 0.966 \\
\hline$A(2-1-3)$ & 108.7 & $A(2-1-3)$ & 107.0 \\
\hline$A(2-1-10)$ & 111.5 & $A(2-1-18)$ & 111.5 \\
\hline$A(1-2-4)$ & 110.3 & $A(1-2-4)$ & 110.2 \\
\hline$A(1-2-5)$ & 110.9 & $A(1-2-5)$ & 110.7 \\
\hline$A(1-2-6)$ & 106.5 & $A(1-2-6)$ & 108.1 \\
\hline$A(3-1-10)$ & 108.8 & $A(3-1-18)$ & 108.6 \\
\hline$A(1-3-7)$ & 106.0 & $A(1-3-7)$ & 105.3 \\
\hline$A(1-3-12)$ & 111.2 & $A(1-3-8)$ & 110.4 \\
\hline$A(1-3-15)$ & 111.2 & $A(1-3-9)$ & 111.0 \\
\hline$A(4-2-5)$ & 107.2 & $A(4-2-5)$ & 108.0 \\
\hline$A(4-2-6)$ & 109.2 & $A(4-2-6)$ & 112.3 \\
\hline$A(5-2-6)$ & 112.8 & $A(5-2-6)$ & 107.6 \\
\hline$A(2-6-7)$ & 101.8 & $A(2-6-7)$ & 104.1 \\
\hline$A(2-6-11)$ & 112.5 & $A(2-6-10)$ & 112.1 \\
\hline$A(2-6-13)$ & 112.7 & $A(2-6-16)$ & 106.9 \\
\hline$A(7-3-12)$ & 109.5 & $A(7-3-8)$ & 109.3 \\
\hline$A(7-3-15)$ & 113.9 & $A(7-3-9)$ & 111.9 \\
\hline$A(3-7-6)$ & 103.3 & $A(3-7-6)$ & 102.4 \\
\hline$A(3-7-8)$ & 109.5 & $A(3-7-11)$ & 109.3 \\
\hline$A(3-7-9)$ & 113.2 & $A(3-7-12)$ & 113.7 \\
\hline$A(12-3-15)$ & 105.2 & $A(8-3-9)$ & 108.9 \\
\hline$A(3-15-16)$ & 125.2 & $A(3-9-13)$ & 125.0 \\
\hline$A(3-15-17)$ & 112.1 & $A(3-9-14)$ & 112.2 \\
\hline$A(7-6-11)$ & 112.6 & $A(7-6-10)$ & 111.6 \\
\hline$A(7-6-13)$ & 106.4 & $A(7-6-16)$ & 112.4 \\
\hline$A(6-7-8)$ & 109.8 & $A(6-7-11)$ & 109.9 \\
\hline$A(6-7-9)$ & 112.4 & $A(6-7-12)$ & 112.7 \\
\hline$A(11-6-13)$ & 110.3 & $A(10-6-16)$ & 109.6 \\
\hline$A(6-13-14)$ & 109.5 & $A(6-16-17)$ & 109.1 \\
\hline$A(8-7-9)$ & 108.5 & $A(11-7-12)$ & 108.6 \\
\hline$A(16-15-17)$ & 122.6 & $A(13-9-14)$ & 122.7 \\
\hline$A(15-17-18)$ & 107.2 & $A(9-14-15)$ & 107.3 \\
\hline
\end{tabular}




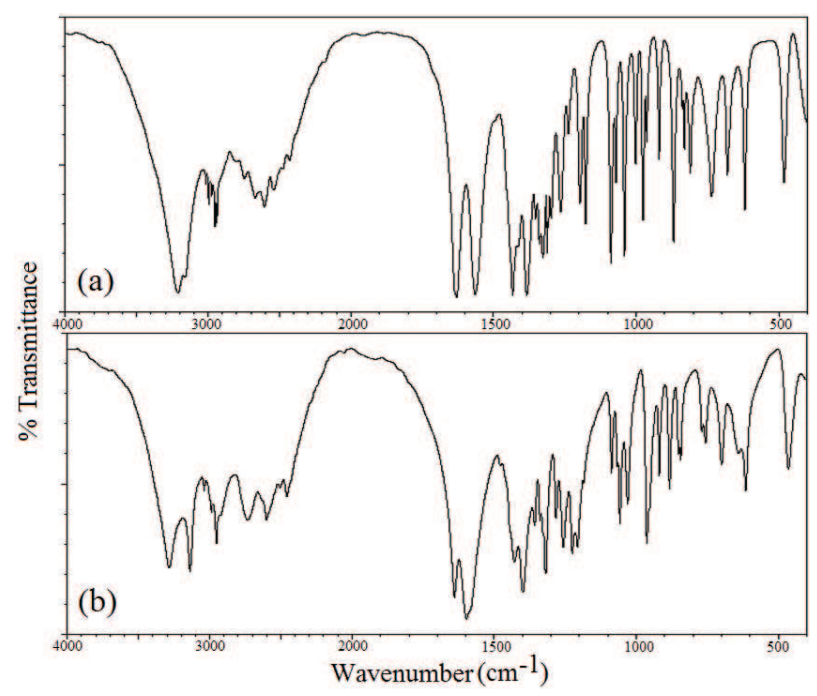

Figure 2: FTIR spectra of 4-hydroxy-l-proline (a) cis conformer (b) trans conformer.

values. The FTIR spectra of both conformers are displayed in Fig. 2. In Fig. 3, we have shown a correlation between calculated and experimental frequencies. In both cases, the correlation coefficients $\left(R^{2}\right)$ are close enough to unity, ensuring the reliability of our computations. Furthermore, $R^{2}$ value for trans-4HLP $(0.99886)$ is higher than that for cis-4HLP (0.99608), showing a better correlation in case of trans conformer.

Table 2 and 3 list vibrational frequencies, IR intensity and corresponding assignments including PED for cis-4HLP and trans-4HLP, respectively. Note that PED less than $10 \%$ are not included in the assignment. We divide the whole spectra into three regions for the sake of simplicity of discussion.

\subsubsection{Spectral region above $1800 \mathrm{~cm}^{-1}$}

In this region, nearly all modes correspond to the stretching vibrations. The highest frequency corresponds to $\mathrm{OH}$ stretching with PED 100\%, polarized perpendicular to the ring are obtained at $3691 \mathrm{~cm}^{-1}$ and $3690 \mathrm{~cm}^{-1}$ in cis- and trans-4HLP, respectively which is associated to $-\mathrm{OH}$ group, whereas, at $3624 \mathrm{~cm}^{-1}$ in cis and $3618 \mathrm{~cm}^{-1}$ in trans conformer associated to $-\mathrm{COOH}$ group. This difference may due to intra-molecular hydrogen bond which is stronger in case of trans-4HLP, $13 \mathrm{O} \cdots 15 \mathrm{H}(2.23 \AA)$ than cis conformer $16 \mathrm{O} \cdots 18 \mathrm{H}(2.29 \AA)$. At $3421 \mathrm{~cm}^{-1}$, pure $\mathrm{NH}$ stretching mode polarized along $3 \mathrm{C}-15 \mathrm{C}$ in cis form and corresponding mode in trans conformer lies at $3409 \mathrm{~cm}^{-1}$. Furthermore, in cis-4HLP, $\mathrm{CH}$ stretching vibrations polarized along a plane contain $7 \mathrm{C}$ making $45^{\circ}$ with plane of ring is calculated at $2973 \mathrm{~cm}^{-1}$ with PED of $82 \%$, agreeing well with the experimental value of $2976 \mathrm{~cm}^{-1}$. Similarly, corresponding mode of vibration polarized along 3C-8H in trans-4HLP lies at $2997 \mathrm{~cm}^{-1}$ having FTIR value of $2986 \mathrm{~cm}^{-1}$. Other $\mathrm{CH}$ modes lying at $2966 \mathrm{~cm}^{-1}$ (90\% PED) and $2925 \mathrm{~cm}^{-1}$ (84\% PED) in cis conformer match well with the experimental values, $2976 \mathrm{~cm}^{-1}$ and $2938 \mathrm{~cm}^{-1}$, respectively. Moreover, 


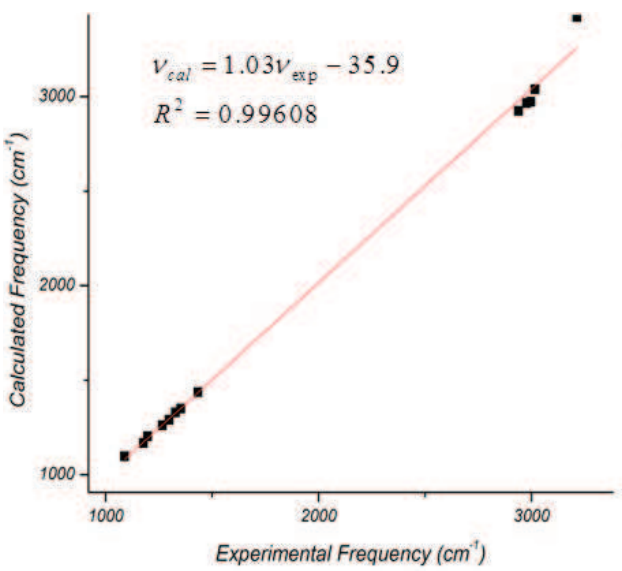

(a)

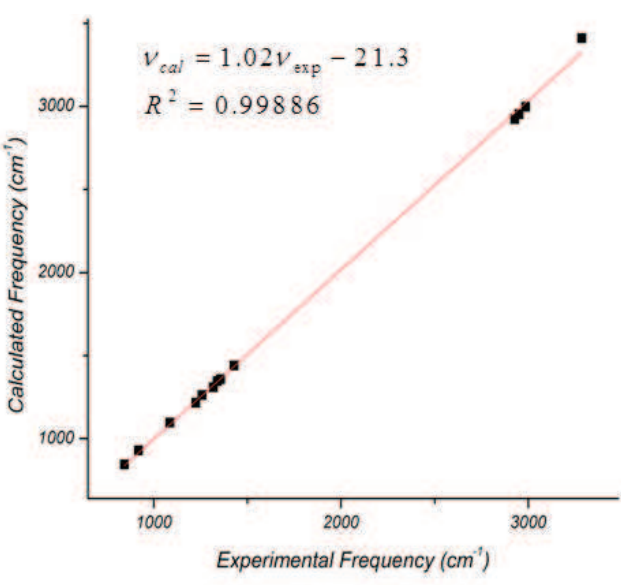

(b)

Figure 3: Correlation between calculated (scaled) frequency $\left(v_{\text {cal }}\right)$ and experimental frequency $\left(v_{\exp }\right)$ for cis (a) and trans (b) conformers of 4-hydroxy- $l$-proline.

corresponding modes in trans form, scaled at $2953 \mathrm{~cm}^{-1}$ (65\% PED) and $2922 \mathrm{~cm}^{-1}$ (85\% PED) are seen at $2951 \mathrm{~cm}^{-1}$ and $2928 \mathrm{~cm}^{-1}$ in FTIR spectrum.

\subsubsection{Spectral region $1800-1000 \mathrm{~cm}^{-1}$}

Carbonyl absorptions are very sensitive as both the carbon and oxygen atoms of $-\mathrm{COOH}$ group move during the vibration, having nearly equal amplitude. In cis-4HLP, a very intense band due to $\mathrm{CO}$ stretching polarized along $1 \mathrm{~N}-10 \mathrm{H}$ direction is calculated at 1746 $\mathrm{cm}^{-1}$ with PED of $78 \%$. A mixing of vibrational modes obtained at $1322 \mathrm{~cm}^{-1}$, polarized along the plane of ring, corresponds to out of plane $\mathrm{CNH}$ bending $(21 \%)$ and $\mathrm{CH} 2$ rocking $(12 \%)$ which is in agreement with the experimental one (see Table 2). At $1291 \mathrm{~cm}^{-1}$, a moderately intense mode corresponding to the mixing of $\mathrm{CHC}$ wagging (29\%), CC stretching (12\%) and $\mathrm{CHO}$ out of plane bending (10\%) matches well with experimental value of $1298 \mathrm{~cm}^{-1}$. Furthermore, the mixing of $-\mathrm{COOH}$ group deformation with $\mathrm{CH} 2$ rocking mode and CN stretching mode are calculated at $1262 \mathrm{~cm}^{-1}$ and $1098 \mathrm{~cm}^{-1}$ against the experimental bands at $1266 \mathrm{~cm}^{-1}$ and $1089 \mathrm{~cm}^{-1}$, respectively.

In trans-4HLP, like its cis form, a very intense band due to $\mathrm{CO}$ stretching polarized along $1 \mathrm{~N}-18 \mathrm{H}$ direction is calculated at $1747 \mathrm{~cm}^{-1}$ with a PED of $79 \%$. At $1345 \mathrm{~cm}^{-1}$, a mixing of out of plane deformation of $-\mathrm{COOH}$ group (20\%), CC stretching (16\%) and CN stretching $(10 \%)$ corresponds to the experimental band at $1339 \mathrm{~cm}^{-1}$. Other significant modes calculated at $1262 \mathrm{~cm}^{-1}$ and $1096 \mathrm{~cm}^{-1}$ correspond to the mixing of $-\mathrm{COOH}$ group deformation with in plane $\mathrm{NH}$ bending and $\mathrm{CCN}$ deformation with $\mathrm{CN}$ stretching agree well with the experiment values of $1258 \mathrm{~cm}^{-1}$ and $1087 \mathrm{~cm}^{-1}$. 
Table 2: Proposed assignments and potential energy distribution (PED) for vibrational modes of cis-4-hydroxyl-proline calculated at B3LYP/6-31+G(d,p) level.

\begin{tabular}{|c|c|c|c|c|}
\hline $\begin{array}{l}\text { Calc. Freq. } \\
\left(\mathrm{cm}^{-1}\right)\end{array}$ & $\begin{array}{r}\text { Scaled Freq. } \\
\left(\mathrm{cm}^{-1}\right)\end{array}$ & $\begin{aligned} \text { Int. } \\
\text { (a.u.) }\end{aligned}$ & $\begin{array}{r}\text { FTIR Freq. } \\
\left(\mathrm{cm}^{-1}\right)\end{array}$ & $\begin{array}{l}\text { Assignments } \\
(\mathrm{PED} \geq 10 \%)\end{array}$ \\
\hline 3826 & 3691 & 26.3 & & $v(\mathrm{O} 13 \mathrm{H} 14)(100)$ \\
\hline 3757 & 3624 & 67.5 & & $v(\mathrm{O} 17 \mathrm{H} 18)(100)$ \\
\hline 3546 & 3421 & 31.8 & 3212 & $v(\mathrm{~N} 1 \mathrm{H} 10)(100)$ \\
\hline 3150 & 3039 & 5.0 & 3016 & $v(\mathrm{C} 7 \mathrm{H} 9)(84)+v(\mathrm{C} 7 \mathrm{H} 8)(16)$ \\
\hline 3082 & 2973 & 32.7 & 2996 & $v(\mathrm{C} 7 \mathrm{H} 8)(82)+v(\mathrm{C} 7 \mathrm{H} 9)(15)$ \\
\hline 3075 & 2966 & 31.6 & 2976 & $v(\mathrm{C} 2 \mathrm{H} 5)(90)$ \\
\hline 3032 & 2925 & 88.7 & 2938 & $v(\mathrm{C} 6 \mathrm{H} 11)(84)+v(\mathrm{C} 2 \mathrm{H} 4)(12)$ \\
\hline 3018 & 2911 & 27.8 & & $v(\mathrm{C} 3 \mathrm{H} 12)(97)$ \\
\hline 3010 & 2904 & 35.8 & & $v(\mathrm{C} 2 \mathrm{H} 4)(77)+v(\mathrm{C} 6 \mathrm{H} 11)(13)$ \\
\hline 1810 & 1746 & 296 & & $v(\mathrm{C} 15 \mathrm{O} 16)(78)$ \\
\hline 1509 & 1455 & 0.5 & & $\rho(\mathrm{CH} 2)(85)+\rho(\mathrm{CH} 2)(11)$ \\
\hline 1489 & 1436 & 7.1 & 1434 & $\rho(\mathrm{CH} 2)(84)+\rho(\mathrm{CH} 2)(12)$ \\
\hline 1481 & 1428 & 12.0 & & $\delta(\mathrm{C} 2 \mathrm{H} 10 \mathrm{~N} 1)(71)$ \\
\hline 1400 & 1350 & 4.3 & 1353 & $\delta(\mathrm{H} 11 \mathrm{O} 13 \mathrm{C} 6)(36)+\delta(\mathrm{C} 6 \mathrm{H} 14 \mathrm{O} 13)(12)+v(\mathrm{C} 6 \mathrm{C} 7)(10)$ \\
\hline 1378 & 1329 & 22.5 & 1328 & $\omega(\mathrm{N} 1 \mathrm{H} 4 \mathrm{C} 2)(23)+\delta(\mathrm{H} 11 \mathrm{O} 13 \mathrm{C} 6)(12)+\omega(\mathrm{C} 6 \mathrm{H} 8 \mathrm{C} 7)(10)$ \\
\hline 1371 & 1322 & 38.5 & & $\omega(\mathrm{N} 1 \mathrm{H} 4 \mathrm{C} 2)(21)+\rho(\mathrm{CH} 2)(12)$ \\
\hline 1339 & 1291 & 25.8 & 1298 & $\begin{array}{l}\omega(\mathrm{C} 2 \mathrm{H} 11 \mathrm{C} 6)(29)+v(\mathrm{C} 2 \mathrm{C} 6)(12) \\
\quad+\delta(\mathrm{H} 11 \mathrm{O} 13 \mathrm{C} 6)(10)+\delta(\mathrm{C} 6 \mathrm{H} 14 \mathrm{O} 13)(10)\end{array}$ \\
\hline 1327 & 1280 & 0.4 & & $\omega(\mathrm{C} 6 \mathrm{H} 8 \mathrm{C} 7)(42)+\omega(\mathrm{N} 1 \mathrm{H} 4 \mathrm{C} 2)(20)$ \\
\hline 1309 & 1262 & 20.4 & 1266 & $\rho(\mathrm{CH} 2)(34)+\delta(\mathrm{C} 15 \mathrm{H} 18 \mathrm{O} 17)(22)+\rho(\mathrm{CH} 2)(15)$ \\
\hline 1281 & 1235 & 24.3 & & $\omega(\mathrm{CH} 2)(29)+\tau(\mathrm{N} 1 \mathrm{H} 4 \mathrm{C} 2)(15)+\tau(\mathrm{N} 1 \mathrm{H} 12 \mathrm{C} 3)(13)$ \\
\hline 1247 & 1203 & 0.6 & 1198 & $\begin{array}{l}\tau(\mathrm{N} 1 \mathrm{H} 4 \mathrm{C} 2)(24)+\omega(\mathrm{CH} 2)(20) \\
\quad+\tau(\mathrm{N} 1 \mathrm{H} 12 \mathrm{C} 3)(14)+\tau(\mathrm{C} 6 \mathrm{H} 8 \mathrm{C} 7)(11)\end{array}$ \\
\hline 1210 & 1167 & 6.1 & 1178 & $\tau(\mathrm{C} 6 \mathrm{H} 8 \mathrm{C} 7)(32)+\tau(\mathrm{N} 1 \mathrm{H} 4 \mathrm{C} 2)(12)$ \\
\hline 1177 & 1135 & 75.4 & & $v(\mathrm{C} 15 \mathrm{O} 17)(21)+\delta(\mathrm{C} 15 \mathrm{H} 18 \mathrm{O} 17)(18)+v(\mathrm{~N} 1 \mathrm{C} 2)(12)$ \\
\hline 1173 & 1131 & 46.3 & & $\delta(\mathrm{C} 6 \mathrm{H} 14 \mathrm{O} 13)(25)+\omega(\mathrm{C} 2 \mathrm{H} 11 \mathrm{C} 6)(15)+\tau(\mathrm{C} 2 \mathrm{H} 11 \mathrm{C} 6)(13)$ \\
\hline 1139 & 1098 & 227.5 & 1089 & $v(\mathrm{~N} 1 \mathrm{C} 2)(27)+v(\mathrm{~N} 1 \mathrm{C} 2)(14)+\delta(\mathrm{C} 15 \mathrm{H} 18 \mathrm{O} 17)(12)$ \\
\hline 1098 & 1059 & 29.8 & & $\begin{array}{l}v(\mathrm{C} 6 \mathrm{O} 13)(20)+\tau(\mathrm{C} 6 \mathrm{H} 8 \mathrm{C} 7)(14) \\
\quad+v(\mathrm{~N} 1 \mathrm{C} 2)(14)+\delta \operatorname{inp}(\mathrm{R})(13)\end{array}$ \\
\hline 1060 & 1022 & 2.2 & & $\tau(\mathrm{N} 1 \mathrm{H} 4 \mathrm{C} 2)(15)+v(\mathrm{C} 3 \mathrm{C} 7)(11)+v(\mathrm{C} 6 \mathrm{O} 13)(11)$ \\
\hline 983 & 948 & 7.2 & & $v(\mathrm{C} 3 \mathrm{C} 7)(24)+v(\mathrm{~N} 1 \mathrm{C} 2)(13)$ \\
\hline 977 & 942 & 28.7 & & $\delta \operatorname{inp}(\mathrm{R})(20)+v(\mathrm{C} 6 \mathrm{O} 13)(13)+v(\mathrm{C} 6 \mathrm{C} 7)(12)+v(\mathrm{C} 3 \mathrm{C} 7)(11)$ \\
\hline 961 & 927 & 14.8 & & $\delta \operatorname{inp}(\mathrm{R})(30)+v(\mathrm{~N} 1 \mathrm{C} 2)(17)+v(\mathrm{C} 6 \mathrm{O} 13)(14)+v(\mathrm{C} 2 \mathrm{C} 6)(13)$ \\
\hline 932 & 899 & 5.7 & & $v(\mathrm{C} 6 \mathrm{C} 7)(24)+v(\mathrm{C} 3 \mathrm{C} 15)(16)+v(\mathrm{C} 2 \mathrm{C} 6)(10)$ \\
\hline 870 & 839 & 15.1 & & $v(\mathrm{C} 2 \mathrm{C} 6)(28)+v(\mathrm{C} 6 \mathrm{O} 13)(17)+\delta \operatorname{inp}(\mathrm{R})(14)$ \\
\hline 825 & 795 & 30.2 & & $\delta \operatorname{inp}(\mathrm{R})(37)+\delta \operatorname{oop}(\mathrm{C} 15 \mathrm{O} 16)(18)+\rho(\mathrm{CH} 2)(12)$ \\
\hline 798 & 769 & 26.3 & & $\delta$ oop $(\mathrm{H} 10 \mathrm{C} 2 \mathrm{C} 3)(29)+v(\mathrm{C} 3 \mathrm{C} 7)(17)$ \\
\hline 777 & 749 & 4.0 & & $v(\mathrm{C} 3 \mathrm{C} 15)(13)+\delta \mathrm{inp}(\mathrm{R})(22)+v(\mathrm{C} 6 \mathrm{C} 7)(11)+v(\mathrm{C} 6 \mathrm{O} 13)(10)$ \\
\hline 722 & 696 & 71.8 & & $\delta \mathrm{oop}(\mathrm{H} 10 \mathrm{C} 2 \mathrm{C} 3)(33)+v(\mathrm{~N} 1 \mathrm{C} 2)(12)$ \\
\hline 681 & 657 & 84.2 & & $\begin{array}{l}\delta \operatorname{inp}(\mathrm{R})(31)+\delta \mathrm{oop}(\mathrm{C} 15 \mathrm{O} 16)(20) \\
\quad+\tau(\mathrm{C} 3 \mathrm{C} 15)(13)+\delta \mathrm{oop}(\mathrm{H} 10 \mathrm{C} 2 \mathrm{C} 3)(10)\end{array}$ \\
\hline 608 & 586 & 33.9 & & $\delta(\mathrm{O} 16 \mathrm{O} 17 \mathrm{C} 3)(40)+\tau(\mathrm{C} 3 \mathrm{C} 15)(28)$ \\
\hline 549 & 529 & 41.5 & & $\tau(\mathrm{C} 3 \mathrm{C} 15)(40)+\delta \mathrm{oop}(\mathrm{C} 15 \mathrm{O} 16)(15)$ \\
\hline 454 & 438 & 17.1 & & $\delta(\mathrm{O} 16 \mathrm{O} 17 \mathrm{C} 3)(29)+\delta(\mathrm{O} 16 \mathrm{C} 15 \mathrm{C} 3)(16)$ \\
\hline
\end{tabular}

\subsubsection{Spectral region below $1000 \mathrm{~cm}^{-1}$}

As expected, the ring torsions along with wagging modes appear in this low frequency region. For cis-4HLP, a mixing of modes appears at $696 \mathrm{~cm}^{-1}$, corresponding to out of plane $\mathrm{CCH}$ bending (33\%) and $\mathrm{CN}$ stretching $(12 \%)$. A polarized mode of vibration with polarization vector along $\mathrm{CN}$ bond occurs at $657 \mathrm{~cm}^{-1}$, corresponding to deformations of ring and out of plane $-\mathrm{COOH}$ with the PED of $31 \%$ and $16 \%$, respectively. For trans 
Table 3: Proposed assignments and potential energy distribution (PED) for vibrational modes of trans-4hydroxy-l-proline calculated at B3LYP/6-31+G(d,p) level.

\begin{tabular}{|c|c|c|c|c|}
\hline $\begin{array}{l}\text { Calc. Freq. } \\
\left(\mathrm{cm}^{-1}\right)\end{array}$ & $\begin{array}{l}\text { Scaled Freq. } \\
\left(\mathrm{cm}^{-1}\right)\end{array}$ & $\begin{aligned} \text { Int. } \\
\text { (a.u.) }\end{aligned}$ & $\begin{array}{r}\text { FTIR Freq. } \\
\left(\mathrm{cm}^{-1}\right)\end{array}$ & $\begin{array}{l}\text { Assignments } \\
(\mathrm{PED} \geq 10 \%)\end{array}$ \\
\hline 3825 & 3690 & 29.0 & & $v(\mathrm{O} 16 \mathrm{H} 17)(100)$ \\
\hline 3750 & 3618 & 73.0 & & $v(\mathrm{O} 14 \mathrm{H} 15)(100)$ \\
\hline 3534 & 3409 & 15.7 & 3286 & $\nu(\mathrm{N} 1 \mathrm{H} 18)(99)$ \\
\hline 3112 & 3009 & 26.3 & & $v(\mathrm{C} 7 \mathrm{H} 12)(74)+v(\mathrm{C} 7 \mathrm{H} 11)(23)$ \\
\hline 3107 & 2997 & 21.0 & 2986 & $v(\mathrm{C} 2 \mathrm{H} 4)(65)+v(\mathrm{C} 2 \mathrm{H} 5)(33)$ \\
\hline 3070 & 2961 & 12.6 & & $v(\mathrm{C} 3 \mathrm{H} 8)(91)$ \\
\hline 3061 & 2953 & 40.1 & 2951 & $v(\mathrm{C} 2 \mathrm{H} 5)(65)+v(\mathrm{C} 2 \mathrm{H} 4)(30)$ \\
\hline 3049 & 2941 & 33.3 & & $v(\mathrm{C} 7 \mathrm{H} 11)(63)+v(\mathrm{C} 7 \mathrm{H} 12)(16)+v(\mathrm{C} 6 \mathrm{H} 10)(11)$ \\
\hline 3029 & 2922 & 29.3 & 2928 & $v(\mathrm{C} 6 \mathrm{H} 10)(85)+v(\mathrm{C} 7 \mathrm{H} 11)(11)$ \\
\hline 1811 & 1747 & 300.7 & & $v(\mathrm{C} 9 \mathrm{O} 13)(79)$ \\
\hline 1505 & 1452 & 3.6 & & $\delta(\mathrm{H} 4 \mathrm{H} 5 \mathrm{C} 2)(81)+\tau(\mathrm{H} 18 \mathrm{C} 2)(14)$ \\
\hline 1493 & 1440 & 1.6 & 1430 & $v(\mathrm{~N} 1 \mathrm{C} 2)(38)+\delta(\mathrm{H} 11 \mathrm{H} 12 \mathrm{C} 7)(17)+\delta \operatorname{inp}(\mathrm{R})(10)$ \\
\hline 1439 & 1388 & 9.0 & & $\delta(\mathrm{H} 11 \mathrm{H} 12 \mathrm{C} 7)(56)+v(\mathrm{~N} 1 \mathrm{C} 2)(17)$ \\
\hline 1409 & 1359 & 12.0 & 1358 & $\delta(\mathrm{H} 10 \mathrm{O} 61 \mathrm{C} 6)(41)+\omega(\mathrm{NH})(11)$ \\
\hline 1395 & 1345 & 92.6 & 1339 & $\begin{array}{l}\delta(\mathrm{C} 12 \mathrm{C} 6 \mathrm{O} 11)(20)+v(\mathrm{~N} 1 \mathrm{C} 2)(16) \\
\quad+v(\mathrm{C} 3 \mathrm{C} 9)(10)+\delta \operatorname{inp}(\mathrm{R})(10)\end{array}$ \\
\hline 1357 & 1309 & 3.9 & 1319 & $\omega(\mathrm{NH})(46)$ \\
\hline 1345 & 1297 & 16 & & $\omega(\mathrm{CH} 2)(29)+\omega(\mathrm{N} 1 \mathrm{H} 8 \mathrm{C} 3)(12)+\tau(\mathrm{C} 3 \mathrm{H} 8 \mathrm{~N} 1)(11)$ \\
\hline 1324 & 1277 & 9.6 & & $\omega(\mathrm{N} 1 \mathrm{H} 8 \mathrm{C} 3)(25)+\tau(\mathrm{C} 3 \mathrm{H} 8 \mathrm{~N} 1)(18)+\mathrm{s}(\mathrm{C} 6 \mathrm{C} 2 \mathrm{H} 10)(15)$ \\
\hline 1309 & 1262 & 27.4 & 1258 & $\begin{array}{l}\delta \operatorname{inp}(\mathrm{R})(18)+\delta(\mathrm{C} 12 \mathrm{C} 6 \mathrm{O} 11)(17) \\
\quad+\delta(\mathrm{C} 12 \mathrm{C} 6 \mathrm{O} 11)(11)+\delta \operatorname{inp}(\mathrm{H} 18 \mathrm{~N} 1)(11)\end{array}$ \\
\hline 1262 & 1217 & 15.3 & 1226 & $\tau(\mathrm{C} 2 \mathrm{~N} 1 \mathrm{H} 4)(42)$ \\
\hline 1260 & 1215 & 33.4 & 1216 & $\omega(\mathrm{CH} 2)(22)+\delta(\mathrm{C} 12 \mathrm{C} 6 \mathrm{O} 11)(20)$ \\
\hline 1226 & 1182 & 43.6 & & $\delta \operatorname{inp}(\mathrm{H} 18 \mathrm{~N} 1)(35)+\delta \mathrm{oop}(\mathrm{N} 1 \mathrm{H} 18)(15)+v(\mathrm{~N} 1 \mathrm{C} 2)(13)$ \\
\hline 1195 & 1152 & 5.0 & & $\begin{array}{l}\omega(\mathrm{C} 6 \mathrm{C} 2 \mathrm{H} 10)(20)+\tau(\mathrm{C} 6 \mathrm{C} 2 \mathrm{H} 10)(15) \\
\quad+\tau(\mathrm{C} 7 \mathrm{C} 3 \mathrm{H} 11)(14)+\delta(\mathrm{C} 6 \mathrm{H} 17 \mathrm{O} 16)(11)\end{array}$ \\
\hline 1163 & 1122 & 211.4 & & $v(\mathrm{C} 9 \mathrm{O} 14)(32)+\delta(\mathrm{C} 12 \mathrm{C} 6 \mathrm{O} 11)(24)+\delta(\mathrm{C} 12 \mathrm{C} 6 \mathrm{O} 11)(10)$ \\
\hline 1137 & 1096 & 100.7 & 1087 & $v(\mathrm{~N} 1 \mathrm{C} 3)(40)+\delta(\mathrm{N} 1 \mathrm{C} 3 \mathrm{C} 7)(31)+v(\mathrm{~N} 1 \mathrm{C} 2)(15)$ \\
\hline 1085 & 1046 & 15.0 & & $\begin{array}{l}\delta \operatorname{inp}(\mathrm{H} 18 \mathrm{~N} 1)(17)+\delta \operatorname{inp}(\mathrm{R})(13) \\
\quad+v(\mathrm{C} 3 \mathrm{C} 7)(11)+v(\mathrm{~N} 1 \mathrm{C} 3)(11)\end{array}$ \\
\hline 1044 & 1007 & 43.2 & & $\delta \operatorname{inp}(\mathrm{H} 18 \mathrm{~N} 1)(35)+\delta \mathrm{oop}(\mathrm{N} 1 \mathrm{H} 18)(15)+v(\mathrm{~N} 1 \mathrm{C} 2)(13)$ \\
\hline 1039 & 1002 & 5.7 & & $v(\mathrm{C} 6 \mathrm{C} 7)(31)+\delta \operatorname{inp}(\mathrm{H} 18 \mathrm{~N} 1)(10)$ \\
\hline 984 & 949 & 4.0 & & $\begin{array}{l}v(\mathrm{C} 3 \mathrm{C} 9)(24)+\delta \text { inp }(\mathrm{H} 18 \mathrm{~N} 1)(12) \\
\quad+v(\mathrm{~N} 1 \mathrm{C} 2)(12)+v(\mathrm{C} 3 \mathrm{C} 7)(10)\end{array}$ \\
\hline 964 & 930 & 48.1 & 920 & $\begin{array}{l}v(\mathrm{C} 2 \mathrm{C} 6)(21)+\delta \operatorname{inp}(\mathrm{R})(19)+v(\mathrm{C} 6 \mathrm{O} 16)(14) \\
\quad+v(\mathrm{~N} 1 \mathrm{C} 3)(10)+\tau(\mathrm{H} 18 \mathrm{C} 2)(10)\end{array}$ \\
\hline 930 & 897 & 10.8 & & $v(\mathrm{C} 3 \mathrm{C} 7)(16)+\delta \operatorname{inp}(\mathrm{R})(12)$ \\
\hline 875 & 844 & 2.4 & 844 & $\begin{array}{l}\delta \operatorname{inp}(\mathrm{R})(20)+\delta \operatorname{inp}(\mathrm{H} 18 \mathrm{~N} 1)(16) \\
\quad+v(\mathrm{C} 3 \mathrm{C} 7)(11)+v(\mathrm{~N} 1 \mathrm{C} 3)(11)\end{array}$ \\
\hline 841 & 811 & 5.6 & & $\begin{array}{l}v(\mathrm{C} 2 \mathrm{C} 6)(22)+\delta \operatorname{inp}(\mathrm{H} 18 \mathrm{~N} 1)(17) \\
\quad+v(\mathrm{C} 6 \mathrm{C} 7)(12)+v(\mathrm{C} 6 \mathrm{O} 16)(10)\end{array}$ \\
\hline 787 & 759 & 134.2 & & $\delta$ oop $(\mathrm{N} 1 \mathrm{H} 18)(59)+\delta \operatorname{inp}(\mathrm{H} 18 \mathrm{~N} 1)(23)$ \\
\hline 738 & 712 & 31.7 & & $\omega(\mathrm{C} 9 \mathrm{C} 3)(34)+\delta \mathrm{inp}(\mathrm{H} 18 \mathrm{~N} 1)(13)+\delta \mathrm{oop}(\mathrm{N} 1 \mathrm{H} 18)(10)$ \\
\hline 715 & 689 & 13.8 & & $\delta \mathrm{oop}(\mathrm{N} 1 \mathrm{H} 18)(26)+\tau(\mathrm{H} 18 \mathrm{C} 2)(19)$ \\
\hline 658 & 634 & 28.3 & & $\delta(\mathrm{N} 1 \mathrm{C} 3 \mathrm{C} 7)(34)+\rho(\mathrm{O} 13 \mathrm{C} 3 \mathrm{C} 9)(28)+\delta \operatorname{inp}(\mathrm{H} 18 \mathrm{~N} 1)(12)$ \\
\hline 613 & 591 & 49.5 & & $\begin{array}{l}\tau(\mathrm{O} 14 \mathrm{C} 9)(26)+\delta(\mathrm{N} 1 \mathrm{C} 3 \mathrm{C} 7)(24) \\
\quad+\delta \operatorname{inp}(\mathrm{R})(16)+\delta \operatorname{inp}(\mathrm{H} 18 \mathrm{~N} 1)(10)\end{array}$ \\
\hline 593 & 572 & 30.0 & & $\tau(\mathrm{O} 14 \mathrm{C} 9)(24)+\delta \operatorname{inp}(\mathrm{H} 18 \mathrm{~N} 1)(19)+\delta \operatorname{inp}(\mathrm{R})(12)+\delta(\mathrm{N} 1 \mathrm{C} 3 \mathrm{C} 7)(10)$ \\
\hline 450 & 434 & 25.4 & & $\delta \operatorname{inp}(R)(26)+\rho(O 13 C 3 C 9)(13)+\delta \operatorname{inp}(R)(12)$ \\
\hline 425 & 410 & 10.1 & & $\begin{array}{l}\tau(\mathrm{C} 6 \mathrm{C} 2 \mathrm{H} 10)(33)+\tau(\mathrm{H} 18 \mathrm{C} 2)(16) \\
\quad+\omega(\mathrm{C} 6 \mathrm{C} 2 \mathrm{H} 10)(13)+\delta \mathrm{inp}(\mathrm{R})(10)\end{array}$ \\
\hline
\end{tabular}

Types of vibrations: $v$-stretching, $\rho$-rocking, $\omega$-wagging, $\tau$-twisting, $\delta$-deformation,

$\delta$ inp-in plane ring bending, $\delta$ oop-out of plane ring bending, $\tau$ - ring torsion. 
conformer, a mixing of modes appears at $689 \mathrm{~cm}^{-1}$ which is corresponding to out of plane $\mathrm{NH}$ bending (26\%) and $\mathrm{CH}$ torsion (19\%). The deformation of $-\mathrm{COOH}$ group mixed with in plane $\mathrm{NH}$ bending is obtained at $634 \mathrm{~cm}^{-1}$.

\subsection{Electronic properties}

Total electronic energies (including zero point energy), energy related parameters and dipole moments of cis and trans conformers of 4HLP are collected in Table 4. Trans conformer is merely $0.027 \mathrm{eV}$ higher in energy than cis-4HLP. Ionization potential $(I)$ and electron affinity $(A)$ of molecules are calculated within the framework of Koopmans' theorem, which describes $I$ and $A$ as the negative of energy eigen-values of the highest occupied molecular orbital (HOMO) and lowest unoccupied molecular orbital (LUMO). The energy difference between HOMO and LUMO results in an energy gap $\left(E_{g a p}\right)$ which is an important factor for analyzing the chemical stability of molecular species.

Table 4: Electronic parameters of cis- and trans-4-hydroxy-l-proline calculated at B3LYP/6-31+G(d,p). Refer to the text for symbols.

\begin{tabular}{|c|c|c|}
\hline Parameters $^{a}$ & cis-4-hydroxy-l-proline & trans-4-hydroxy-l-proline \\
\hline Total energy (a.u.) & -476.2604 & -476.2594 \\
\hline$I(\mathrm{eV})$ & 6.35 & 6.50 \\
\hline$A(\mathrm{eV})$ & 0.38 & 0.47 \\
\hline$E_{g a p}(\mathrm{eV})$ & 5.97 & 6.03 \\
\hline$\mu$ (Debye) & 2.06 & 3.17 \\
\hline
\end{tabular}

$I$ and $A$ values of trans-4HLP are slightly larger than those for its cis counterpart (see Table 4). This may suggest that trans conformer is relatively more stable against addition or removal of an electron. This fact is further supported by its higher $E_{g a p}$ as compared to cis-4HLP. More interestingly, the dipole-moment $(\mu)$ of trans-4HLP exceeds to that of cis-4HLP by 1.11 Debye. The higher dipole-moment of trans conformer results due to the fact that the groups attached to the ring lie in different planes which increase the polarity, unlike cis-4HLP in which both groups lie in the same plane.

\section{Conclusions}

Using density functional theory calculations, we have discussed the structural, vibrational and electronic properties of 4-hydroxy-l-proline in cis and trans conformations. Detailed assignments to the all normal modes up to $450 \mathrm{~cm}^{-1}$ has been offered and potential energy distribution along with direction of polarization has also been discussed. The calculated vibrational frequencies have been found in better agreement with corresponding FTIR spectra. Furthermore, we have noticed that trans conformer is relatively more stable and polar as compared to its cis counterpart. 
Acknowledgments. AKS thanks Council of Scientific and Industrial Research (CSIR), New Delhi, India for providing a research fellowship. The Central Facility for Computational Research (CFCR), University of Lucknow is gratefully acknowledged.

\section{References}

[1] K. L. Gorres and R. T. Raines, Crit. Rev. Biochem. Mol. Biol. 45 (2010) 106.

[2] P. Szpak, J. Archaeol. Sci. 38 (2011) 3358.

[3] D. L. Nelson and M. M. Cox, Lehninger's Principles of Biochemistry, 4th Edition (Freeman and Company, New York, 2005).

[4] J. Brinckmann, H. Notbohm, and P. K. Müller, Collagen, Topics Curr. Chem. 247 (Springer, Berlin, 2005).

[5] G. I. Cassab, Ann. Rev. Plant Physiol. Plant Mol. Biol. 49 (1998) 281.

[6] Wheeless' Textbook of Orthopaedics, available from http://www.wheelessonline.com/ortho/pagets_disease.

[7] R. J. Xavier and P. Dinesh, Spectrochim. Acta A 128 (2014) 54.

[8] M. J. Frisch, G. W. Trucks, H. B. Schlegel, et al., Gaussian 09, Revision B.1, Gaussian (Wallingford, UK, 2010).

[9] A. D. Becke, J. Chem. Phys. 98 (1993) 5648.

[10] C. Lee, W. Yang and R. G. Parr, Phys. Rev. B, 37 (1988) 785.

[11] A. K. Srivastava and N. Misra, Canadian J. Chem. 92 (2014) 234.

[12] A. K. Srivastava and N. Misra, Commun. Comput. Chem. 1 (2013) 328.

[13] A. K. Srivastava, B. Narayana, B. K. Sarojini, and N. Misra, Indian J. Phys. 88 (2014) 547.

[14] A. K. Srivastava, A. K. Pandey, B. Narayana, B. K. Sarojini, P. S. Nayak, and N. Misra, J. Theor. Chem. 2014 (2014) 125841.

[15] J. M. L. Martin, V. Alsenoy, C. V. Alsenoy, Gar2ped (University of Antwerp, 1995).

[16] I. M. Alecu, J. Zheng, Y. Zhao, and D. G. Truhlar, J. Chem. Theory Comput. 6 (2010) 2872.

[17] Available from http://sdbs.db.aist.go.jp (National Institute of Advanced Industrial Science and Technology, accessed Jan. 2014).

[18] T. F. Koetzle, M. S. Lehmann, and W. C. Hamilton, Acta Crystallogr. B 29 (1973) 231. 